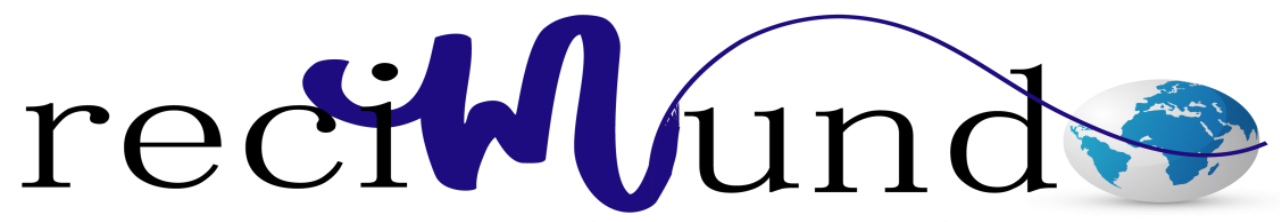

Revista Científica Mundo de la Investigación y el Conocimiento

Glubis Gomez Pelaez ${ }^{\text {a; }}$ Hugo Luna Rodriguez ${ }^{\text {b; }}$ Ana Maria Flores Luna ${ }^{\mathrm{c}}$

Estado nutricional y hábitos alimentarios de los adultos que acuden a consulta externa del dispensario norte del IESS Guayaquil, período de enero - junio del 2011

Revista Científica Mundo de la Investigación y el Conocimiento. Vol. 2 núm., 1, febrero, ISSN: 2588-073X, 2018, pp. 172-189

DOI: 10.26820/recimundo/2.1.2018.172-189

Editorial Saberes del Conocimiento

Recibido: 05/12/2017 Aceptado: 10/02/2018

a. Universidad de Guayaquil; gomezp@ug.edu.ec

b. Universidad de Guayaquil; hugo.lunar@ug.edu.ec

c. Profesional Independiente; anitaflores1984@gmail.com 


\title{
Estado nutricional y hábitos alimentarios de los adultos que acuden a consulta externa del dispensario norte del IESS Guayaquil, período de enero - junio del
}

Vol. 2, núm. 1., (2018)

Glubis Gomez Pelaez; Hugo Luna Rodriguez; Ana Maria Flores Luna

\begin{abstract}
RESUMEN
Un problema importante y de actualidad para la salud pública lo constituyen los trastornos nutricionales que evidencian un incremento acelerado en la edad adulta, motivo por el cual se realizó la investigación, con el objetivo de determinar el estado nutricional y los hábitos alimentarios de los adultos que acudieron a la consulta del Dispensario Norte del IESS Guayaquil de enero a junio del 2011, así como diseñar una propuesta de medidas preventivas basadas en la educación nutriológica continuada. El universo y muestra fue de 360 pacientes que acudieron a la consulta de acuerdo a los criterios de inclusión, se realizó un estudio descriptivo y transversal de tipo prospectivo, para este fin se tomó medidas antropométricas, peso y talla obteniéndose con estos dos parámetros el índice de masa corporal, también se les realizó una encuesta basada en la preferencia alimentaria, por último se correlacionaron los datos obteniéndose como resultados que la mayoría de los adultos jóvenes masculinos tuvieron algún grado de sobrepeso y obesidad, mientras que los adultos medios femeninos se encontraron en mayor porcentaje en normo peso, la mayoría de adultos jóvenes masculinos tuvieron preferencia por alimentos ricos en grasa y carbohidratos, los adultos medios femeninos en cambio tuvieron una dieta más equilibrada con menos preferencia por el consumo de alimentos ricos en grasas y carbohidratos. Las conclusiones a las que se llegó fueron que el mayor consumo de grasas y carbohidratos constituyó un factor importante en la incidencia de sobrepeso y obesidad en el grupo de adultos jóvenes masculinos, motivo por el cual se recomendó la conformación de una comisión encargada del control, fomento y promoción del estado nutricional de los adultos, así como del tratamiento de los trastornos nutricionales que se presenten en los individuos sanos y enfermos, educándolos en nutrición para que sustenten una alimentación adecuada y por ende una vida saludable y productiva, disminuyendo la incidencia y prevalencia de las enfermedades crónico degenerativas y lograr disminuir la morbi-mortalidad de este grupo etario.
\end{abstract}

Palabras clave: Alimentación, nutrición, sobrepeso y obesidad. 


\title{
Estado nutricional y hábitos alimentarios de los adultos que acuden a consulta externa del dispensario norte del IESS Guayaquil, período de enero - junio del 2011
}

Vol. 2, núm. 1., (2018)

Glubis Gomez Pelaez a; Hugo Luna Rodriguez b; Ana Maria Flores Luna c

\begin{abstract}
An important and topical health public problem constitute what nutritional disorders that show an increase accelerated in the adult age, reason for which was carried out the research, with the objective of determining the nutritional status and dietary habits of adults who attended the consultation of the Northern dispensary of the Guayaquil IESS of January to June 2011 as well as design a proposal of preventive measures based on continuous nutriologica education. The universe and sample was of 360 patients who attended the consultation according to the inclusion criteria, a descriptive and transversal study of prospective type for this purpose was taken anthropometric measurements, weight and height obtained with these two parameters the body mass index, was also conducted a survey based on food preference, finally were correlated data obtained as results that most male young adults had some degree of envelope weight and obesity, while female means adults were found in higher percentage in normo weight, the majority of male young adults took preference for foods high in fat and carbohydrates, the female average adults instead had a more balanced diet with fewer preference by the consumption of foods rich in fats and carbohydrates. The conclusions which it reached were that higher consumption of fats and carbohydrates was an important factor in the incidence of envelope weight and obesity, in the Group of young male adults, reason why it was recommended the formation of a Commission to control building and promotion of nutritional status of adults as well as the treatment of nutritional disorders presented in healthy.
\end{abstract}

Keywords: Feeding, nutrition, weight, obesity. 


\section{Estado nutricional y hábitos alimentarios de los adultos que acuden a consulta externa del dispensario norte del IESS Guayaquil, período de enero - junio del}

Vol. 2, núm. 1., (2018)

Glubis Gomez Pelaez; Hugo Luna Rodriguez; Ana Maria Flores Luna

\section{Introducción.}

El desarrollo de un país depende de diversos factores, uno de gran importancia es su capacidad productiva, que se sustenta en la salud de sus habitantes, pues existe una evidente relación entre la salud y la nutrición del individuo, siendo esta última esencial para la supervivencia.

La Organización Mundial de la Salud (OMS) declaró que el mejoramiento de la salud es esencial para que se adelante hacia el logro de los demás objetivos de desarrollo del milenio ${ }^{1}$.

Es precisamente en la salud donde impacta la problemática de la nutrición, porque actualmente existen millones de personas que carecen de una alimentación adecuada. La nutrición a lo largo del curso de la vida es una de las principales determinantes de la salud, del desempeño físico y mental, y de la productividad ${ }^{2}$.

Ciertos de los trastornos que los individuos padecen y consultan con los médicos se deben a que los alimentos que consumen actualmente no reúnen los nutrimentos suficientes para satisfacer las necesidades diarias. Los componentes celulares y tisulares sufren un continuo desgaste y deben ser reemplazados continuamente para mantener la homeostasis que permita la buena salud del individuo, pues de lo contrario darán origen a las enfermedades ${ }^{3}$.

La alimentación de los adultos en general tienen una relación importante con sus hábitos alimentarios, pues los malos hábitos desencadenarán u ocasionarán trastornos nutricionales que 


\section{Estado nutricional y hábitos alimentarios de los adultos que acuden a consulta externa del dispensario norte del IESS Guayaquil, período de enero - junio del 2011}

Vol. 2, núm. 1., (2018)

Glubis Gomez Pelaez a; Hugo Luna Rodriguez b; Ana Maria Flores Luna c

van desde la desnutrición a la obesidad, ambas constituyen problemas de salud mundial porque inciden en la población en diferentes aspectos sin distinguir estratos sociales. La malnutrición es una afectación gradual en el estado nutricional, que en estados más avanzados ocasiona deterioro de las capacidades y complicaciones clínicas ${ }^{4}$.

La malnutrición es propia de los países en vías de desarrollo y el sobrepeso y obesidad se presentan tanto en países desarrollados como en vías de desarrollo, la obesidad ha tenido un incremento importante en estos últimos años debido probablemente al incremento en la disponibilidad de alimentos altamente energéticos, ricos en grasas saturadas trans y azúcares, acompañada de una disminución de la actividad física. La obesidad infantil se asocia a una mayor probabilidad de muerte prematura y discapacidad en la edad adulta ${ }^{5}$.

No existe en el Ecuador un estudio importante sobre hábitos alimentarios o estado nutricional de los adultos, solo programas de intervención para mejorar el estado nutricional del adulto mayor a través de la entrega de alimentos fortificados considerando solo la dimensión socio-económica.

Es importante realizar este estudio, para determinar el estado nutricional y los hábitos alimentarios en los adultos, así como relacionar las diferencias existentes en las etapas de las edades adultas y jóvenes. Se podrá establecer cuál es la realidad en nuestro medio y poder establecer las medidas estratégicas oportunas y necesarias, realizando las recomendaciones para mejorar la salud y por ende la calidad de vida, previniendo el padecimiento de enfermedades crónicas degenerativas. 


\section{Estado nutricional y hábitos alimentarios de los adultos que acuden a consulta externa del dispensario norte del IESS Guayaquil, período de enero - junio del}

de la salud y mejoramiento de la calidad de vida, siendo la alimentación el mayor factor modificable de las enfermedades crónicas degenerativas.

Un problema importante de actualidad y que constituye un problema para la salud pública son los trastornos nutricionales existentes y crecientes a nivel mundial, es decir, de los países del primer mundo como los países en vías de desarrollo, existen pues claras evidencias de la relación entre los hábitos alimentarios y el estado nutricional de los individuos, esto validado a través de estudios realizados a nivel mundial.

En el Ecuador no se han realizado investigaciones importantes para confirmar y determinar cuál es la realidad en nuestro medio en el campo de la nutrición. Las diferentes instituciones universitarias, gubernamentales, no gubernamentales, y sociedades científicas poco o nada han realizado en este campo, sobre todo en el grupo etario de los adultos, y de éstos, aún menos en los adultos jóvenes y maduros; algo se ha hecho en los adultos mayores, donde existen medidas de intervención basadas en las dimensiones socio-económicas más que propiamente las basadas en investígaciones nutricionales.

Se evidencia el incremento acelerado de los trastornos nutricionales en la edad adulta que van desde la desnutrición hasta el sobrepeso y obesidad, debido a los malos hábitos alimentarios y al desconocimiento de cómo alimentarse adecuadamente, situación que condiciona la aparición creciente también de enfermedades crónicas y el aumento de la morbi-mortalidad. 


\section{Estado nutricional y hábitos alimentarios de los adultos que acuden a consulta externa del dispensario norte del IESS Guayaquil, período de enero - junio del 2011}

Vol. 2, núm. 1., (2018)

Glubis Gomez Pelaez a; Hugo Luna Rodriguez b; Ana Maria Flores Luna c

El propósito de esta investigación fue establecer y sugerir una adecuada alimentación a través de la educación nutriológica continuada con miras a modificar los malos hábitos alimentarios a largo plazo.

El objetivo de la presente investigación es determinar el estado nutricional, los hábitos alimentarios y propuestas de medidas preventivas de los adultos que asistieron a la consulta del dispensario norte IESS de Guayaquil, de enero a junio del 2011.

\section{Metodología.}

Lugar de la investigación

El Dispensario Norte del IESS se encuentra ubicado en la subparroquia Tarqui Este, subdivisión de la parroquia urbana Tarqui del sector norte de la ciudad de Guayaquil, se localiza en una zona clasificada como Zona Industrial. Los límites de esta parroquia inician en las faldas de los cerros Santa Ana y del Carmen y abarcan todo el sector norte hasta el límite con el río Guayas, y por el lado Sur va desde la intersección de las avenidas Quito y Nueve de Octubre hasta el perímetro urbano del puerto que, según la Municipalidad, llega al km 26 de la vía a la costa. Esta parroquia avanza incluso hasta el km 28 de la vía a Daule.

El Dispensario Norte del IESS se encuentra ubicado en la Cdla. Sta. Adriana Km 6.5 de la Av. Juan Tanca Marengo de la Subparroquia Tarqui Este. 


\section{Estado nutricional y hábitos alimentarios de los adultos que acuden a consulta externa del dispensario norte del IESS Guayaquil, período de enero - junio del}

Vol. 2, núm. 1., (2018)

Glubis Gomez Pelaez; Hugo Luna Rodriguez; Ana Maria Flores Luna

\section{Universo}

La población estuvo constituida por todos los pacientes que acudieron a la consulta externa de medicina y cirugía general del Dispensario Norte IESS en la ciudad de Guayaquil.

\section{Procedimiento de la investigación}

Para poder realizar la presente investigación fue necesaria la autorización, colaboración y consentimiento de los directores ejecutivo y técnico del Dispensario Norte IESS, jefes de los servicios de medicina y cirugía de los que se programó captar los pacientes, de los médicos de la consulta externa que colaboraron para la investigación y de los pacientes que acudieron a la consulta externa de medicina y cirugía general que cumplieron con los criterios de inclusión. La investigación se realizó de enero a junio del 2011, en los pacientes seleccionados se aplicó las encuestas tipo frecuencia de alimentos para conocer sus hábitos alimentarios en lo que se refiere a las preferencias de consumo de carbohidratos, grasas o proteínas, y se determinó su estado nutricional por el método antropométrico a través de la talla y peso, estableciendo el índice de masa corporal. Con estos datos se agruparon los individuos con bajo peso, normo peso, sobrepeso y obesidad, correlacionándolos con el sexo, edad y sus hábitos del tipo de preferencias alimentarias para establecer el vínculo existente de tipo causa efecto.

\section{Técnica de análisis de datos}

De los datos recolectados se realizó un análisis cuantitativo, determinando el índice de masa corporal a través de la talla y peso obtenidos, se agruparan estos datos de acuerdo al grupo de edades de los adultos sean jóvenes o maduros, se estableció el sexo al que corresponden, 


\section{Estado nutricional y hábitos alimentarios de los adultos que acuden a consulta externa del dispensario norte del IESS Guayaquil, período de enero - junio del 2011}

Vol. 2, núm. 1., (2018)

Glubis Gomez Pelaez a; Hugo Luna Rodriguez b; Ana Maria Flores Luna c

además de las encuestas obtenidas se establecieron las preferencias alimentarias, y se correlacionaron todos los datos obtenidos, con el objeto de determinar qué porcentaje de individuos tienen un índice de masa corporal definido y relacionado a su grupo etario, sexo, y preferencia alimentaria.

Manejo de la investigación

Se realizó un informe final, haciendo conocer los resultados obtenidos de acuerdo a los objetivos e hipótesis planteadas, los aspectos más relevantes de la investigación, y la difusión de los mismos considerando los aspectos éticos, también se estableció las conclusiones y recomendaciones de acuerdo a estos resultados obtenidos.

Aspectos legales y éticos

Se tomaron en consideración los aspectos éticos y legales, tales como el solicitar el permiso a las autoridades del dispensario, profesionales médicos y pacientes, a estos últimos se solicitó a través de su consentimiento informado, donde de manera explícita se dio a conocer el propósito de la investigación, el tipo de participación que tendrían, la confidencialidad y anonimato de la información adquirida, los potenciales beneficios individuales y colectivos de los resultados de la investigación. 
Estado nutricional y hábitos alimentarios de los adultos que acuden a consulta externa del dispensario norte del IESS Guayaquil, período de enero - junio del

2011

Vol. 2, núm. 1., (2018)

Glubis Gomez Pelaez; Hugo Luna Rodriguez; Ana Maria Flores Luna

\section{Resultados.}

ESTADO NUTRICIONAL DE LOS ADULTOS QUE ACUDIERON A LA CONSULTA

DEL DISPENSARIO NORTE IESS. ENERO A JUNIO DEL 2011.

\begin{tabular}{|l|c|c|}
\hline GRUPO DE ADULTOS & NUMERO & PORCENTAJE \\
\hline Adultos jóvenes & 244 & $68 \%$ \\
\hline Adultos maduros & 116 & $32 \%$ \\
\hline TOTAL & $\mathbf{3 6 0}$ & $\mathbf{1 0 0 \%}$ \\
\hline
\end{tabular}

Cuadro \# 1. Porcentaje de adultos estudiados

De un universo de 360 pacientes adultos estudiados que asistieron a consulta, 244 de estos adultos que representan el 68\%, son adultos jóvenes y que constituyen la mayoría, mientras que los 116 adultos restantes que corresponden al 32\% son adultos medios o maduros. Esta concurrencia mayoritaria se debe a la ubicación geográfica de esta unidad de salud que abarca una gran zona industrial y de comercio donde la mayor fuerza laboral la constituyen los adultos jóvenes. Y por ende tienen mayor exposición y predisposición a padecer quebrantamiento en su salud.

\begin{tabular}{|l|c|c|}
\hline SEXO & NÚMERO & PORCENTAJE \\
\hline Masculino & 201 & $56 \%$ \\
\hline Femenino & 159 & $44 \%$ \\
\hline TOTAL & $\mathbf{3 6 0}$ & $\mathbf{1 0 0 \%}$ \\
\hline
\end{tabular}

Gráfico \# 2. Porcentaje de los adultos estudiados según su sexo 


\section{Estado nutricional y hábitos alimentarios de los adultos que acuden a consulta externa del dispensario norte del IESS Guayaquil, período de enero - junio del 2011}

Vol. 2, núm. 1., (2018)

Glubis Gomez Pelaez a; Hugo Luna Rodriguez b; Ana Maria Flores Luna c

De un universo de 360 adultos objeto de estudio su distribución en relación al género resulto la siguiente, al sexo masculino le corresponde el 56\%, representado por los 201 adultos, mientras que al sexo femenino le corresponde el $44 \%$ constituidos por los 159 adultos restantes.

En general, de todo el conglomerado de adultos estudiados existe mayoría del sexo masculino, en relación al sexo femenino que está en minoría.

\begin{tabular}{|l|c|c|c|}
\hline ADULTOS & MASCULINOS & FEMENINOS & TOTAL \\
\hline Adultos jóvenes & $136(56 \%)$ & $108(44 \%)$ & $244(100 \%)$ \\
\hline Adultos maduros & $65(56 \%)$ & $51(44 \%)$ & $116(100 \%)$ \\
\hline TOTAL & $\mathbf{2 0 1}$ & $\mathbf{1 5 9}$ & $360(100 \%)$ \\
\hline
\end{tabular}

\section{Gráfico \# 3. Porcentaje de adultos estudiados en relación a su sexo.}

De un total de 244 adultos jóvenes, 136 (56\%) corresponden al sexo masculino y 108 (44\%) restantes al sexo femenino. Del total de 116 adultos maduros o medios, 65 (56\%) son del sexo masculino y los $51(44 \%)$ restantes son del sexo femenino.

Se observa que existen más adultos masculinos jóvenes y medios en el estudio en relación a los adultos femeninos jóvenes y medios. También se observa que a pesar de tener una diferencia numérica los adultos masculinos de los femeninos jóvenes y medios, existe una proporción igualitaria de los adultos masculinos jóvenes y medios (56\%), lo mismo que en los adultos femeninos jóvenes y medios (44\%). 
Estado nutricional y hábitos alimentarios de los adultos que acuden a consulta externa del dispensario norte del IESS Guayaquil, período de enero - junio del

2011

Vol. 2, núm. 1., (2018)

Glubis Gomez Pelaez; Hugo Luna Rodriguez; Ana Maria Flores Luna

\begin{tabular}{|l|c|c|}
\hline \multicolumn{1}{|c|}{ IMC } & NÚMERO & PORCENTAJE \\
\hline Peso insuficiente & 0 & $0 \%$ \\
\hline Normo peso & 153 & $43 \%$ \\
\hline Sobrepeso grado I & 39 & $11 \%$ \\
\hline Sobrepeso grado II & 88 & $24 \%$ \\
\hline Obesidad I & 55 & $15 \%$ \\
\hline Obesidad II & 17 & $5 \%$ \\
\hline Obesidad III & 6 & $2 \%$ \\
\hline Obesidad IV & 2 & $1 \%$ \\
\hline TOTAL & $\mathbf{3 6 0}$ & $\mathbf{1 0 0 \%}$ \\
\hline
\end{tabular}

Gráfico \# 4. Porcentaje del índice de masa corporal de los adultos estudiados.

De los 360 adultos estudiados, el 43\% (153 adultos) se encuentran con normo peso, mientras que el 57\% (207 adultos) se encuentran con sobrepeso y obesidad. De estos 207 adultos el 61,35\% (127) se encuentran con sobrepeso, mientras que el 38,64\% (80 adultos) se encuentran con obesidad. De los adultos con obesidad III que son el 2\% (6) y obesidad IV que son el $1 \%$ (2) fueron los referidos de otros servicios como endocrinología y medicina interna al servicio de cirugía general para ingresar al programa de cirugía bariátrica. 
Estado nutricional y hábitos alimentarios de los adultos que acuden a consulta externa del dispensario norte del IESS Guayaquil, período de enero - junio del 2011

Vol. 2, núm. 1., (2018)

Glubis Gomez Pelaez a; Hugo Luna Rodriguez b; Ana Maria Flores Luna c

CONSUMO DE ALIMENTOS DE LOS ADULTOS QUE ACUDIERON A LA CONSULTA DEL DISPENSARIO NORTE IESS. ENERO A JUNIO DEL 2011.

\begin{tabular}{|l|c|c|l|}
\hline $\begin{array}{l}\text { GRUPO DE } \\
\text { ADULTOS }\end{array}$ & NÚMERO & PORCENTAJE & $\begin{array}{l}\text { FRECUENCIA DE } \\
\text { CONSUMO DE ALIMENTOS }\end{array}$ \\
\hline Adultos jóvenes & 161 & $78 \%$ & Grasas y Carbohidratos \\
\hline Adultos maduros & 46 & $22 \%$ & Grasas y Carbohidratos \\
\hline TOTAL & $\mathbf{2 0 7}$ & $\mathbf{1 0 0 \%}$ & \\
\hline
\end{tabular}

Gráfico \# 5. Porcentaje de la frecuencia de consumo de alimentos de los adultos estudiados en relación al grupo de edad

Del total de 360 adultos estudiados, 207 adultos (58\%) tienen un consumo frecuente de alimentos con preferencia por un mayor consumo de grasas y carbohidratos, mientras que los 153 adultos restantes (42\%) tienen una dieta más equilibrada en cuanto al consumo frecuente de alimentos de grasas y carbohidratos. De los 207 adultos (58\%) con desequilibrio en su dieta, 161 (78\%) son adultos jóvenes y los 46 restantes (22\%) son adultos maduros.

Existe mayor cantidad de adultos jóvenes con desequilibrio en su dieta que en los adultos medios debido a su modo de vida, ingiriendo mayor cantidad de comidas rápidas y con alto valor energético, pues este grupo etario demuestra poco interés por alimentarse adecuadamente y cuidar su aspecto físico. 
Estado nutricional y hábitos alimentarios de los adultos que acuden a consulta externa del dispensario norte del IESS Guayaquil, período de enero - junio del

2011

Vol. 2, núm. 1., (2018)

Glubis Gomez Pelaez; Hugo Luna Rodriguez; Ana Maria Flores Luna

\begin{tabular}{|l|c|c|c|c|c|c|}
\hline IMC & MASCULINO & & FEMININO & & TOTAL & \\
\hline & Numero & $\%$ & Numero & $\%$ & Numero & $\%$ \\
\hline Peso insuficiente & 0 & 0 & 0 & 0 & 0 & 0 \\
\hline Normo peso & 70 & 34,82 & 83 & 52,20 & 153 & 42,5 \\
\hline Sobrepeso I & 24 & 11,94 & 15 & 9,43 & 39 & 10,83 \\
\hline Sobrepeso II & 56 & 27,86 & 32 & 20,12 & 88 & 24,44 \\
\hline Obesidad I & 34 & 16,91 & 21 & 13,20 & 55 & 15,27 \\
\hline Obesidad II & 11 & 5,47 & 6 & 3,77 & 17 & 4,72 \\
\hline Obesidad III & 4 & 1,99 & 2 & 1,25 & 6 & 1,66 \\
\hline Obesidad IV & 2 & 0.99 & 0 & 0 & 0 & 0 \\
\hline TOTAL & 201 & 100 & 159 & 100 & 360 & 100 \\
\hline
\end{tabular}

\begin{tabular}{|l|c|c|l|}
\hline IMC & MASCULINO & FEMENINO & TOTAL \\
\hline Normo peso & $70(34,82 \%)$ & $83(52,2 \%)$ & $\mathbf{1 5 3 ( 4 2 , 5 \% )}$ \\
\hline Sobrepeso & $80(39,8 \%)$ & $47(29,55 \%)$ & $\mathbf{1 2 7 ( 3 5 , 2 7 \% )}$ \\
\hline Obesidad & $51(25,37 \%)$ & $29(18,23 \%)$ & $\mathbf{8 0 ( 2 2 , 2 2} \%)$ \\
\hline TOTAL & $\mathbf{2 0 1 ( 1 0 0 \% )}$ & $\mathbf{1 5 9}(\mathbf{1 0 0} \%)$ & $\mathbf{3 6 0 ( 1 0 0 \% )}$ \\
\hline
\end{tabular}

Cuadro \# 6. Porcentaje del índice de masa corporal de los adultos estudiados en relación al sexo.

De los 201 adultos masculinos, el 35\% (70 varones) se encontraron con normo peso, mientras el 65\% (131 varones) tuvieron sobrepeso y obesidad. De los 159 adultos femeninos el $52 \%$ (83 mujeres) se encontraron con normo peso y el $48 \%$ (76 mujeres) tuvieron sobrepeso y obesidad. De los 127 adultos con sobrepeso el 62.99\% (80 adultos) fueron del sexo masculino y el 30\% (47 adultos) fueron del sexo femenino. 


\section{Estado nutricional y hábitos alimentarios de los adultos que acuden a consulta externa del dispensario norte del IESS Guayaquil, período de enero - junio del 2011}

Vol. 2, núm. 1., (2018)

Glubis Gomez Pelaez a; Hugo Luna Rodriguez b; Ana Maria Flores Luna c

De los 80 adultos con obesidad, el 25\% (51 adultos) fueron del sexo masculino y el 18\% (29 adultos) fueron del sexo femenino.

\begin{tabular}{|l|c|c|c|c|c|c|}
\hline \multicolumn{1}{|c|}{ IMC } & \multicolumn{2}{c|}{$\begin{array}{c}\text { ADULTO } \\
\text { JOVEN }\end{array}$} & \multicolumn{2}{c|}{$\begin{array}{c}\text { ADULTO } \\
\text { MEDIO }\end{array}$} & \multicolumn{2}{c|}{ TOTAL } \\
\hline & Número & $\%$ & Número & $\%$ & Número & $\%$ \\
\hline Peso insuficiente & 0 & 0 & 0 & 0 & 0 & 0 \\
\hline Normo peso & 83 & 34,01 & 70 & 60,34 & 153 & 42,5 \\
\hline Sobrepeso I & 30 & 12,29 & 9 & 7,75 & 39 & 10,83 \\
\hline Sobrepeso II & 68 & 27,86 & 20 & 17,24 & 88 & 24,44 \\
\hline Obesidad I & 45 & 18,44 & 10 & 8,62 & 55 & 15,27 \\
\hline Obesidad II & 12 & 4,91 & 5 & 4,31 & 17 & 4,72 \\
\hline Obesidad III & 4 & 1,63 & 2 & 1,72 & 6 & 1,66 \\
\hline Obesidad IV & 2 & 0,81 & 0 & 0 & 2 & 0,55 \\
\hline TOTAL & 244 & & 116 & & 360 & \\
\hline
\end{tabular}

\begin{tabular}{|l|c|c|c|}
\hline IMC & ADULTO JOVEN & ADULTO MEDIO & TOTAL \\
\hline Normo peso & $83(34 \%)$ & $70(60.34 \%)$ & $153(42.5 \%)$ \\
\hline Sobrepeso & $98(40.16 \%)$ & $29(25 \%)$ & $127(35.27 \%)$ \\
\hline Obesidad & $63(25.81 \%)$ & $17(14.65 \%)$ & $80(22.22 \%)$ \\
\hline TOTAL & $244(100 \%)$ & $116(100 \%)$ & $360(100 \%)$ \\
\hline
\end{tabular}

Cuadro \# 7. Porcentaje del índice de masa corporal de los adultos estudiados en relación al grupo de edad.

De los 244 adultos jóvenes el 34\% (83 adultos jóvenes) se encontraron con normo peso, el $66 \%$ (161 adultos jóvenes) tuvieron sobrepeso y obesidad. De los 116 adultos medios el 60.34\% 


\section{Estado nutricional y hábitos alimentarios de los adultos que acuden a consulta externa del dispensario norte del IESS Guayaquil, período de enero - junio del}

Vol. 2, núm. 1., (2018) Glubis Gomez Pelaez; Hugo Luna Rodriguez; Ana Maria Flores Luna (70 adultos medios) tuvieron normo peso, mientras el 39.65\% (46 adultos medios) se encontraron con sobrepeso y obesidad.

De los adultos con sobrepeso el $40.16 \%$ (98) fueron adultos jóvenes y el 25\% (29) fueron adultos medios, mientras que los adultos con obesidad el 25.81\% (63) fueron adultos jóvenes y el $14.65 \%$ (17) fueron adultos medios.

\begin{tabular}{|l|c|c|c|}
\hline SEXO & NÚMERO & PORCENTAJE & $\begin{array}{l}\text { FRECUENCIA DE CONSUMO DE } \\
\text { ALIMENTOS }\end{array}$ \\
\hline Masculinos & 131 & $63 \%$ & Grasas y Carbohidratos \\
\hline Femeninos & 76 & $37 \%$ & Grasas y Carbohidratos \\
\hline TOTAL & $\mathbf{2 0 7}$ & $\mathbf{1 0 0} \%$ & \\
\hline
\end{tabular}

CUADRO \# 8. Porcentaje de la frecuencia de consumo de alimentos de los adultos estudiados en relación al sexo.

Del 58\% de adultos en relación al sexo y a la frecuencia de consumo de alimentos preferente de grasas y carbohidratos, el 63\% corresponde al sexo masculino que está en mayoría y el 37\% restante a los adultos del sexo femenino.

Existe mayor desorden alimentario en los adultos masculinos que en los adultos femeninos donde es menos frecuente, posiblemente debido a que en este grupo hay mayor interés en el cuidado de su aspecto físico y conservación de la salud. 


\section{Estado nutricional y hábitos alimentarios de los adultos que acuden a consulta externa del dispensario norte del IESS Guayaquil, período de enero - junio del 2011}

Vol. 2, núm. 1., (2018)

Glubis Gomez Pelaez a; Hugo Luna Rodriguez b; Ana Maria Flores Luna c

\section{Conclusiones.}

Se concluyó, que del total de adultos estudiados los adultos jóvenes de sexo masculino presentaron en mayor porcentaje diferentes grados de sobrepeso y obesidad, relacionado con la mayor frecuencia de consumo de grasas y carbohidratos, a diferencia de los adultos medios de sexo femenino que presentaron en mayor porcentaje normo peso, es decir un estado nutricional dentro de parámetros normales en relación a su índice de masa corporal, relacionado a una menor frecuencia de consumo de grasas y carbohidratos en su dieta.

Estos trastornos nutricionales de los grupos de adultos jóvenes masculinos se explicaría por el mayor consumo de grasas y carbohidratos en su dieta diaria, y se pueden entender que el inadecuado hábito alimentario lo han adquirido por diferente circunstancia, Esta situación se da no solo por los hábitos adquirido en sus hogares, sino también, por sus jornadas de trabajo y compartir el tiempo con sus estudios u otra actividad, que los obligan a consumir comidas rápidas; y otros tipos de comidas en sitios no adecuados con alto contenido de grasas y carbohidratos y de escaso valor nutritivo, esto sumado al sedentarismo y desconocimiento de cómo alimentarse adecuadamente.

En lo referente a los adultos medios en ellos existe mayor concienciación de cómo alimentarse de manera sana y los beneficios que se obtiene en el mantenimiento de su salud, por otra parte las féminas tiene mayor preocupación por mantener un peso adecuado relacionado más al aspecto estético que al de salud. 


\section{Estado nutricional y hábitos alimentarios de los adultos que acuden a consulta externa del dispensario norte del IESS Guayaquil, período de enero - junio del}

Vol. 2, núm. 1., (2018)

Glubis Gomez Pelaez; Hugo Luna Rodriguez; Ana Maria Flores Luna

Los objetivos planteados al inicio de este estudio, se cumplieron a través de la recopilación de datos por la valoración antropométrica y la aplicación de encuestas de frecuencia de consumo de alimentos. Se logró cumplir el objetivo general de este estudio, con respecto a determinar el estado nutricional y los hábitos alimentarios de los adultos.

También, se pudo corroborar la hipótesis planteada en la investigación, al determinar que existen alteraciones del estado nutricional en la etapa de adultez y de la relación existente con sus hábitos alimentarios.

\section{Bibliografía.}

1. OMS. Informe sobre la Salud en el Mundo Ginebra: Organización Mundial de la Salud ; 2003.

2. Fox BA, Cameron AG. Ciencia de los alimentos, nutrición y salud México: LIMUSA WILEY; 1992.

3. Martínez Roldán C, Veiga Herreros P, Cobo Sanz J. Evaluación del estado nutricional de un grupo de adultos mayores de 50 años mediante parámetros dietéticos y de composición corporal. Nutrición Hospitalaria. 2011 Oct; 26(5): p. 1081-1090.

4. Bartriña A. Clínicas Españolas de Nutrición Madrid: Trillas; 2005.

5. Casanueva E, Kaufer-Horwitz M, Pérez-Lizaur A. Nutriologiamédica México: Panamericana; 1995. 